\title{
Effect of hyposalivation on mastication and mandibular movements during speech
}

\section{Simone Guimarães Farias Gomes(a) \\ Altair Antoninha Del Bel Cury(b) Renata Cunha Matheus Rodrigues Garcia $^{(b)}$}

(a) Department of Clinics and Preventive Dentistry, Dental School, Federal University of Pernambuco, Recife, PE, Brazil.

(b) Department of Prosthodontics and Periodontogy, Piracicaba Dental School, State University of Campinas, Piracicaba, SP, Brazil.
Declaration of Interests: The authors certify that they have no commercial or associative interest that represents a conflict of interest in connection with the manuscript.

\section{Corresponding author:}

Renata Cunha Matheus Rodrigues Garcia E-mail: regarcia@fop.unicamp.br

Received for publication on Jan 31, 2011 Accepted for publication on May 04, 2011

\begin{abstract}
The aim of this study was to evaluate whether patients with hyposalivation present damaged mastication or mandibular movements during speech. Forty subjects composed 2 groups: control and hyposalivation. Masticatory performance was assessed with a silicon-based artificial material and a 10-sieve method. Mandibular movements during speech were observed with a 3D jaw-tracking device. Data were analyzed with Student's $t$-test or the Mann-Whitney method $(P<0.05)$. The masticatory performance $(\mathrm{mm})$ of control and hyposalivation were $4.40 \pm 0.62$ and $4.74 \pm 1.34$, respectively. Outcomes for speech movements $(\mathrm{mm})$ were as follows: maximum vertical opening amplitude $(10.8 \pm 4.2 ; 9.9 \pm 2.7)$ and displacements to the right $(1.0 \pm 0.8 ; 0.5 \pm 0.6)$, left $(1.8 \pm 1.4$; $2.3 \pm 1.2)$, or anterior-posterior $(2.8 \pm 1.1 ; 2.9 \pm 1.0)$ for control and hyposalivation, respectively. No statistical difference was found between groups for any variable. The results indicated that hyposalivation did not affect masticatory performance or mandibular movements during speech. ClinicalTrials.gov Identifier: NCT01020084.
\end{abstract}

Descriptors: Saliva; Mastication; Mandible; Movement.

\section{Introduction}

The production of sufficient saliva to moisten food and bind it into a bolus is indispensable for good chewing and deglutition, ${ }^{1}$ which are also influenced by food characteristics. Hard and dry foods, for example, require more chewing cycles and longer times in the mouth until swallowing than other foods for sufficient breakdown and enough saliva to form a coherent bolus. ${ }^{2}$ Difficulties in chewing dry foods and swallowing have been reported by $25 \%$ and $29 \%$ of xerostomic patients, respectively. ${ }^{3}$ Additionally, healthy patients submitted to xerostomia induction by the administration of systemic anticholinergic medications display compromised masticatory efficiencies, due to a lack of saliva after chewing natural foods or artificial material., ${ }^{4,5}$

Saliva also plays an important role in speech; one report found that $48 \%$ of patients who complain about xerostomia also report difficulties with communication. ${ }^{3}$ The expiratory air is modified into sound at the larynx. The mandibular and soft tissue movements during speech modify the oral cavity spaces and affect the production of specific phonemes. ${ }^{6}$ Some hyposalivation-inducing drugs might promote oropharingeal dryness, movement changes, and speech alterations. ${ }^{7}$ Although the mandibu- 
lar tridimensional track during communication has been documented, ${ }^{6,8}$ no objective analysis of jaw movements during speech has been made relative to salivary flow.

The relationship of mastication and speech with saliva may be due to the moistening function of saliva $^{9}$ induced by some constituents, particularly mucins. These proteins coat the oral mucosa with a lubrication pellicle, which protects oral surfaces ${ }^{10}$ and promotes flexibility and freedom to the tissues to allow the movements needed for adequate mastication $^{9}$ and communication. ${ }^{6,11,12}$ A lack of or reduction in salivary flow may damage the moistening features of saliva and may lead to difficulties during functions. ${ }^{3}$

The condition of reduced salivary flow is common, and its major causes are dehydration and the use of systemic medications, ${ }^{13,14}$ hundreds of which have the potential to induce xerostomic effects. ${ }^{9,13,15}$ The xerostomic condition may be accompanied by oral side effects, ${ }^{16}$ such as dryness of mouth and lips, ${ }^{17}$ thirst, cheilitis, and an increased damaged, missing, and filled teeth index, ${ }^{16}$ as a consequence of the decreased salivary flow rate. ${ }^{16,17}$ Some potentially xerostomic drugs may also cause common associated mucocutaneous adverse effects, ${ }^{18-20}$ such as dry skin, nose bleeds, and dry eyes. ${ }^{18}$

No study has addressed the possible oral functional alterations caused by the hyposalivation/xerostomia condition in patients who undergo oral treatment with systemic medication. Thus, the aim of this study was to investigate the masticatory performance and mandibular track pattern during speech in subjects with normal and reduced salivary flow rates induced by a systemic drug.

\section{Methodology}

Forty subjects (20 male and 20 female) aged 1627 years participated in the study. To be included, the subjects had to be fully dentate, except for the absence of the third molars or bicuspids removed for prior orthodontic treatment. Other inclusion criteria included good general health, no history of xerostomia, no signs or symptoms of temporomandibular disorders, no parafunctions, and no history of communication deficits or prior speech-language treatment. Oral examination was assessed by an experienced operator for oral health and severe malocclusion. Subjects presenting caries, periodontal disease, open bite, cross bite, or overjet $>5 \mathrm{~mm}$ were excluded.

Students and staff of Piracicaba Dental School were selected, as were patients undergoing systemic isotretinoin treatment for acne according to medical recommendations. This study was approved by the Ethics Committee of Piracicaba Dental School, University of Campinas (number 063/2006). Written consent was provided by all participants or by their caregivers (in the case of participants $<18$ years old).

Subjects were divided into 2 groups: control, with normal salivary flow rate, and hyposalivation. The hyposalivation was composed of 20 consecutive patients originating from dermatologic clinics who were taking a single dose of isotretinoin daily (0.5$0.7 \mathrm{mg} / \mathrm{kg} / \mathrm{d}$ ) for at least $1 \mathrm{month}$, as prescribed by a dermatologist. All hyposalivation participants were instructed to report mucocutaneous symptoms, such as xerostomia, dry skin, dryness of nose, and dry eyes, after the initiation of treatment with isotretinoin. The participants could not be using saliva substitution or any other kind of intervention to induce salivary flow. The control was composed of subjects matched for age and gender who were not taking any systemic medication.

Unstimulated whole saliva was collected between 7:30 and 10:30 am local time to determine the salivary flow immediately before the mandibular movements during pronunciation of the Brazilian Portuguese Language phonemes and before masticatory evaluations. The saliva sample was collected for 5 min, during which time the subject was instructed to expectorate saliva into a preweighed container. The salivary flow rate was calculated as the difference between the weights of the container before and after expectoration, as a function of time collection.

Assuming a saliva density of $1.0 \mathrm{~g} / \mathrm{mL}$, the weight was divided by 5 and the salivary flow rate was expressed in $\mathrm{mL} / \mathrm{min} .{ }^{21}$ The salivary flow rate was classified in accordance with Dawes, ${ }^{22}$ in which saliva secretion between 0.3 and $0.4 \mathrm{~mL} / \mathrm{min}$ is considered normal and a salivary flow below this rating 
is considered low, characterizing the hyposalivation condition.

Masticatory performance was assessed with a silicon rubber-based artificial test material (Heraeus Kulzer, Hanau, Germany). The silicon was manipulated according to manufacturer`s instructions. It was accommodated into a metallic mould that generated cubes with $5.6-\mathrm{mm}$ edges. After the initial reticulation of the silicon, the cubes were removed from the mould, individually weighed, and placed in an electric stove for total reticulation for $16 \mathrm{~h}$ at $60{ }^{\circ} \mathrm{C} .{ }^{23}$ The cubes were cooled to ambient temperature, disinfected with $2 \%$ glutaraldehyde solution for $30 \mathrm{~min}$, washed, and dried with absorbent paper.

A 17-cube portion weighing approximately $3.4 \mathrm{~g}$ was given to the subject. The subject was instructed to chew the cubes in his/her habitual manner for 20 chewing strokes, which were counted by the operator. The subject then expectorated the fragmented material into a paper filter sitting on a glass container, which was proceeded by several mouth rinses with $200 \mathrm{~mL}$ of water to promote cleanness of the mouth.

Once completely drained, the paper filter was stored in an electrical stove for $25 \mathrm{~min}$ at $80{ }^{\circ} \mathrm{C} .{ }^{24}$ The filter was moved to a system containing 10 sieves (apertures ranging 5.6 to $0.5 \mathrm{~mm}$ ) and placed on a sieving machine (Bertel Indústria Metalúrgica Ltda., Caieiras, Brazil) for the fractioning of the chewed particles. The sieves were individually weighed in a 0.0001-g analytical balance (Bel Engineering S.R., Monza, Italy). Masticatory performance was calculated with the Rosin-Rammler formula, $Q_{w}(X)=100\left(1-2^{-(X / X 50) b}\right)$, in which the median particle size $\left(\mathrm{x}_{50}\right)$ is the theoretical sieve aperture through which $50 \%$ of the weight of the fragmented particles can pass. ${ }^{23}$

To evaluate the pattern of mandibular movements during speech, the maximum values $(\mathrm{mm})$ of the vertical opening amplitude, lateral (right and left) and anterior-posterior deviations were measured with an electromagnetic jaw-tracking device (Myotronics-Noromed Inc., Kent, USA). Each subject was seated on a dental chair, with the Frankfort plane parallel to the ground, and was instructed to avoid head movements. A magnet was temporarily cemented (GC America Inc., Alsip, USA) on the buccal surface of the central lower incisors without interfering with the maximum intercuspal position. A system containing 8 sensors was positioned on the subject's face.

After calibration of the equipment, the subject was told to name each picture in an easily recognizable list of pictures comprising all of the Brazilian Portuguese language phonemes. The subject was instructed to name the pictures sequentially, with no interruptions. ${ }^{6}$ The subject was told to close his/her mouth in the maximal intercuspal position and to name the pictures at a normal conversation rate and volume. The intercuspal position and the picture sequence were demonstrated and practiced before the evaluation.

Both masticatory performance and jaw movements during speech were measured once and at the same session, with a 10 -min interval between tests. The outcomes were analyzed by Student's $t$-test at a 95\% confidence level with SigmaPlot 11.0 software (Systat Software, Inc., Chicago, USA).

\section{Results}

The mean salivary flow rates obtained for the control and hyposalivation were $0.34 \pm 0.04 \mathrm{~mL} /$ $\min$ (range $0.3-0.4 \mathrm{~mL} / \mathrm{min}$ ) and $0.13 \pm 0.07 \mathrm{~mL} /$ min (range $0.03-0.24 \mathrm{~mL} / \mathrm{min}$ ), respectively, indicating well-characterized salivary conditions. ${ }^{22}$ Data from the masticatory performance and oral amplitudes during speech showed no statistical difference $(P>0.05)$ between groups (Table 1$)$.

\begin{tabular}{|c|c|c|c|c|c|c|}
\hline \multirow{4}{*}{$\begin{array}{r}\text { Table } 1 \text { - Masticatory } \\
\text { performance }(\mathrm{mm}) \text { and pattern } \\
\text { of mandibular movements during } \\
\text { speech }(\mathrm{mm}) . \text { Mean } \pm \text { Standard } \\
\text { deviation. }\end{array}$} & \multirow{2}{*}{ Groups } & \multirow{2}{*}{$\begin{array}{l}\text { Masticatory } \\
\text { performance }\end{array}$} & \multirow{2}{*}{$\begin{array}{l}\text { Vertical opening } \\
\text { amplitude }\end{array}$} & \multicolumn{2}{|c|}{ Lateral Deviation } & \multirow{2}{*}{$\begin{array}{c}\text { Antero-Posterior } \\
\text { deviation }\end{array}$} \\
\hline & & & & Right & Left & \\
\hline & Control & $4.4 \pm 0.62$ & $10.8 \pm 4.2$ & $1.0 \pm 0.8$ & $1.8 \pm 1.4$ & $2.8 \pm 1.1$ \\
\hline & Hyposalivation & $4.7 \pm 1.3$ & $9.9 \pm 2.7$ & $0.5 \pm 0.6$ & $2.3 \pm 1.2$ & $2.9 \pm 1.0$ \\
\hline & $P$-value & 0.613 & 0.617 & 0.064 & 0.435 & 0.834 \\
\hline
\end{tabular}




\section{Discussion}

Saliva is responsible for moistening food particles, forming a coherent and slippery bolus to be swallowed, ${ }^{1}$ and lubricating the oral mucosa to facilitate its functions. ${ }^{6,9,11,12}$ The present study was designed to investigate oral functions, such as mastication and mandibular movements during speech, in patients using systemic medications.

Patients with the sensation of dry mouth report difficulties with chewing dry foods and swallowing. ${ }^{1}$ Measurements of the masticatory capacity of individuals after induction of hyposalivation/xerostomia by the administration of systemic anticholinergic drugs indicate decreased masticatory efficiency. ${ }^{4,5}$ Data obtained from the present study did not support these findings, but were consistent with the results of Gomes et al., ${ }^{25}$ who observed, in the same sample, no difference in masticatory efficiency between subjects with hyposalivation, subjects with normal salivary flow rate, and subjects with acute induced hypersalivation.

The different outcomes of these studies could be due to the difference between the samples analyzed: Liedberg and Öwall ${ }^{4}$ and Ishijima et al. ${ }^{5}$ selected subjects who did not take any systemic drug for medical treatment and presented normal salivary flow rates. Drugs with strong xerostomic potential were administered moments before the chewing tests, which abruptly altered the salivary flow and masticatory capacity of the subjects. According to Gomes et al., ${ }^{25}$ acute alteration of the salivary flow rate may cause discomfort or oromotor coordination deficiency during functions, as reported by subjects who were submitted to acute hypersalivation with citric acid. Subjects who participated in the present study had already been under medication with systemic isotretinoin for at least 1 month; they did not suffer acute hyposalivation, and they may have been acclimated to the amount of saliva in their mouths.

Previous studies ${ }^{2,23}$ have addressed the correlation between salivary flow and the number of chewing cycles needed for swallowing many types of foods, such as cake, Melba toast, bread, toast (with or without butter), carrot, peanut, and cheese. The amount of saliva explained only $2 \%$ of the number of chewing cycles needed for swallowing. More- over, a significant, but very weak, negative correlation was found between masticatory performance and salivary flow. This finding suggests that a high salivary flow rate is not necessarily associated with better masticatory efficiency. ${ }^{1,2}$ This information is in accordance with the results of the present study, in which no difference in masticatory performance was detected between the hyposalivation and normal salivary flow groups. Additionally, according to Kaplan et al., ${ }^{26}$ difficulties in mastication and swallowing are more related to advanced salivary gland hypofunction, which is found in patients after head and neck radiation and in patients with Sjögren syndrome.

The present study represents the first attempt to compare the pattern of mandibular movements during speech among subjects with normal and reduced salivary flow as a consequence of systemic therapy. Use of 1 type of medication was preferred for standardization. Isotretinoin is a retinoid commonly used for severe acne treatment. ${ }^{13,15}$ Its systemic use is associated with numerous adverse effects, such as teratogenic potential, alteration of blood parameters, and psychiatric disorders. ${ }^{18-20}$ However, the most common adverse effects associated with oral isotretinoin treatment are mucocutaneous, ${ }^{18-20}$ with an incidence of $100 \% .^{18}$

Many xerostomic subjects complain about difficulties during speech; ${ }^{3}$ however, no study has objectively addressed low salivary flow or how communication may be affected in such patients. Kaplan et al., ${ }^{26}$ analyzed oral functions among subjects with no xerostomia/hyposalivation, xerostomia without salivary gland hypofunction, and salivary grand hypofunction induced by drugs, Sjögren syndrome, head and neck radiation, or idiopathic factors. They reported that alterations in the mucosa and oral function, except for impairment of speech, were more prevalent in salivary gland hypofunction patients. Their data are consistent with the results of the present study, in which the opening amplitude and the lateral and anterior-posterior excursions during the speech of individuals with drug-induced low salivary flow were not altered compared to controls.

The opening amplitudes of the mouth during 
speech, for both the $\mathrm{H}$ and $\mathrm{C}$ groups, are consistent with the results of a prior study that determined normative values for the opening amplitudes of the mouth during speech. ${ }^{6}$ This observation may be due to the presence of the mucin coat on all of the mucosal surfaces of dry mouth patients, including the buccal and labial surfaces. According to Pramanik et al., ${ }^{8}$ even in patients with severe salivary gland hypofunction, the small amounts of saliva produced are sufficient to provide a high molecular weight mucin coating on the oral surfaces. Despite the reduced amount of low molecular weight mucins on the anterior tongue of these patients, its continued presence on the mucosal surfaces may be important to maintain the function of the mucosa in dry mouth, ${ }^{8}$ including the mandibular movements during speech. However, this mucin coat is less hydrated and less mobile due to the reduced mucosal wetness in dry mouth patients, with uncertain effectiveness. ${ }^{8}$

It is well known that the salivary glands are stimulated during mastication due to mechanical and chemical stimuli, with the gustatory stimulation of foods being much more important than the mechanical stimulation from chewing. ${ }^{27}$ We used a silicone-based, and therefore tasteless, artificial material to perform the masticatory task. Regardless, similar amounts of saliva could have been present in the control and hyposalivation groups, due to the mechanical stimulation of the material kneaded during the test.

According to Dawes, ${ }^{28}$ the unstimulated salivary flow rate is rapidly reduced to a basal level after prolonged chewing of flavored chewing gum. Therefore, although the mandibular movements during speech

\section{References}

1. Van Der Bilt A. Assessment of mastication with implications for oral rehabilitation: a review. J Oral Rehabil. 2011 Jan 17; [Epub ahead of print].

2. Engelen L, Fontijn-Tekamp A, van der Bilt A. The influence of product and oral characteristics on swallowing. Arch Oral Biol. 2005 Aug;50(8):739-46.

3. Sreebny LM, Valdini A. Xerostomia. Part I. Relationship to other oral symptoms and salivary gland hypofunction. Oral Surg Oral Med Oral Pathol. 1988 Oct;66(4):451-8. were performed after the chewing test, we believe that the salivary flow had been reduced to the basal values before the speech test was started, because a 10 -min interval was used between the tests. However, the transient increase in salivary secretion of hyposalivation group subjects may have retrieved the wetness of the dry mucin layer over the mucosal surfaces and, consequently, its mobility during function. This explanation may underlie why the control and hyposalivation groups did not present different movement parameters during speech.

Hyposalivation induced by systemic medication therapy with oral isotretinoin did not influence the masticatory performance in this study. However, this finding does not diminish the importance of the reduced salivary flow control for clinicians, who should instruct their patients about the need for frequent ingestion of liquids and salivary stimulation and who should provide oral treatments to prevent dental decay. These measures are intended to reduce the oral signs of dry mouth and the damage caused by lack of saliva, reducing oral deterioration.

\section{Conclusions}

Within the conditions of this study, it can be concluded that hyposalivation induced by systemic medication therapy did not damage masticatory performance or alter the pattern of mandibular movements during speech.

\section{Acknowledgements}

The authors would like to thank São Paulo State Research Foundation (FAPESP), which supported this study (Grant number: 2005/ 03383-1).

\footnotetext{
4. Liedberg B, Öwall B. Masticatory ability in experimentally induced xerostomia. Dysphagia. 1991;6(4):211-3.

5. Ishijima T, Koshino H, Hirai T, Takasaki H. The relationship between salivary secretion rate and masticatory efficiency. J Oral Rehabil. 2004 Jan;31(1):3-6.

6. Bianchini EMG, Andrade CRF. A model of mandibular movements during speech: normative pilot study for the Brazilian Portuguese Language. Cranio. 2006 Jul;24(3):197-206.
} 
7. Abaza MM, Levy S, Hawkshaw MJ, Sataloff RT. Effects of medications on the voice. Otolaryngol Clin North Am. 2007 Oct;40(5):1081-90, viii.

8. Peraire M, Salsench J, Torrent J, Nogueras J, Samsó J. Study of mandibular movements during speech. Cranio. 1990 Oct;8(4):324-31.

9. Sreebny LM. Saliva in health and disease: an appraisal and update. Int Dent J. 2000 Jun;50(3):140-61.

10. Pramanik R, Osailan SM, Challacombe SJ, Urquhart D, Proctor GB. Protein and mucin retention on oral mucosal surfaces in dry mouth patients. Eur J Oral Sci. 2010 Jun;118(3):245-53.

11. Thompson AR. Pharmacological agents with effects on voice. Am J Otolaryngol. 1995 Jan-Feb;16(1):12-8.

12. Smith A, Zelaznik HN. Development of functional synergies for speech motor coordination in childhood and adolescence. Dev Psychobiol. 2004 Jul;45(1):22-33.

13. Sreebny LM, Schwartz SS. A reference guide to drugs and dry mouth - 2nd edition. Gerodontology. 1997 Jul;14(1):33-47.

14. Mese H, Matsuo R. Salivary secretion, taste and hyposalivation. J Oral Rehabil. 2007 Oct;34(10):711-23.

15. Scully C. Drug effects on salivary glands: dry mouth. Oral Dis. 2003 Jul;9(4):165-76.

16. Lupi-Pegurier L, Muller-Bolla M, Fontas E, Ortonne JP. Reduced salivary flow induced by systemic isotretinoin may lead to dental decay. A prospective clinical study. Dermatology. 2007;214(3):221-6.

17. Oikarinen K, Salo T, Kylmäniemi, Palatsi R, Karhunen T, Oikarinen A. Systemic oral isotretinoin therapy and flow rate, $\mathrm{pH}$, and matrix metalloproteinase- 9 activity of stimulated saliva. Acta Odontol Scand. 1995 Dec;53(6):369-71.

18. Kaymak Y, Ilter N. The results and side effects of systemic isotretinoin treatment in 100 patients with acne vulgaris. Dermatol Nurs. 2006 Dec;18(6):576-80.
19. Chivot M. Retinoid therapy for acne: a comparative review. Am J Clin Dermatol. 2005;6(1):13-9.

20. Katsambas A, Papakonstantinou A. Acne: Systemic treatment. Clin Dermatol. 2004 Sep-Oct;22(5):412-8.

21. Richardson CT, Feldman M. Salivary response to food in humans and its effect on gastric acid secretion. Am J Physiol. 1986 Jan;250(1 Pt 1):G85-91.

22. Dawes C. Factors influencing salivary flow rate and composition. In: Edgar M, Dawes C, O’Mullane D. Saliva and Oral Health. 3th ed. London: British Dental Association; 2004. 32-49.

23. Slagter, AP, Bosman F, Van der Bilt A. Comminution of two artificial test foods by dentate and edentulous subjects. J Oral Rehabil. 1993 Mar;20(2):159-76.

24. Omar SM, McEwen JD, Ogston SA. A test for occlusal function: the value of a masticatory efficiency test in the assessment of occlusal function. Br J Orthodont. 1987 Apr;14(2):85-90.

25. Gomes SG, Custódio W, Cury AA, Rodrigues Garcia RCM. Effect of salivary flow rate on masticatory efficiency. Int $\mathrm{J}$ Prosthodont. 2009 Mar-Apr;22(2):168-72.

26. Kaplan I, Zuk-Paz L, Wolff A. Association between salivary flow rates, oral symptoms, and oral mucosal status. Oral Surg Oral Med Oral Pathol Oral Radiol Endod. 2008 Aug;106(2):235-41.

27. Watanabe S, Dawes C. A comparison of the effects of tasting and chewing foods on the flow rate of whole saliva in man. Arch Oral Biol. 1988;33(10):761-4.

28. Dawes C. The unstimulated salivary flow rate after prolonged gum chewing. Arch Oral Biol. 2005 Jun;50(6):561-3. 